\title{
Quantum dot as spin current generator and energy harvester
}

\author{
Barbara Szukiewicz and Karol I. Wysokiński ${ }^{a}$ \\ Institute of Physics, M. Curie-Skłodowska University, Radziszewskiego 10, 20-031 Lublin, Poland
}

Received 27 February 2015 / Received in final form 24 March 2015

Published online 4 May 2015

(C) The Author(s) 2015. This article is published with open access at Springerlink.com

\begin{abstract}
The thermoelectric transport in the device composed of a central nanoscopic system in contact with two electrodes and subject to the external magnetic field of Zeeman type has been studied. The device can support pure spin current in the electrodes and may serve as a source of the temperature induced spin currents with possible applications in spintronics. The system may also be used as an energy harvester. We calculate its thermodynamic efficiency $\eta$ and the power output $P$. The maximal efficiency of the device reaches the Carnot value when the device works reversibly but with the vanishing power. The interactions between carriers diminish the maximal efficiency of the device, which under the constant load drops well below the Carnot limit but may exceed the Curzon-Ahlborn limit. While the effect of intradot Coulomb repulsion on $\eta$ depends on the parameters, the interdot/interlevel interaction strongly diminishes the device efficiency.
\end{abstract}

\section{Introduction}

The desire to find materials and/or structures with a high thermoelectric performance is the driving force of material science and nanotechnology $[1,2]$ and is vital for efficient energy harvesters or refrigerators. The use of the low dimensional systems [3] is one of the ways to engineer devices with high thermoelectric power $S$ and large figure of merit $Z T=G S^{2} T / \kappa$, where $G$ is the conductance, $\kappa$ the thermal conductance and $T$ the temperature. Large thermopower is essential to get high values of $Z T$ as the ratio $\kappa / G T=L$, at least for the Fermi liquid state, is determined by the Lorentz number $L_{0}=\frac{\pi^{2}}{3}\left(\frac{k_{B}}{e}\right)^{2}$. Here $k_{B} / e \approx 82.6 \mu \mathrm{V} / \mathrm{K}$ is the natural unit of thermopower. In the systems which do not obey Wiedemann-Franz law the diminition of the thermal conductivity [4] $\kappa$ and the Lorentz number $L$ is often crucial to achieve large $Z T$.

The importance of $Z T$ follows from the formula

$$
\eta_{Z T}=\eta_{C} \frac{\sqrt{Z T+1}-1}{\sqrt{Z T+1}+1},
$$

relating the figure of merit $Z T$ to the efficiency $\eta$ of the engine working as heat to electric power converter. In the limit of large $Z T$ the device eventually reaches the Carnot efficiency $\eta_{C}$.

In the paper we use simple example of the quantum dot device to calculate its figure of merit, efficiency and the power output at non-equilibrium conditions and find out that the highest power output often corresponds to efficiencies much lower than expected from the above formula. It means that $Z T$, being an important dimensionless

\footnotetext{
${ }^{a}$ e-mail: karol@tytan.umcs.lublin.pl
}

parameter characterizing bulk thermoelectrics [1-3], may not be equally useful in the studies of nanostructures. For example, the figure of merit of the non-interacting quantum dot is infinite [5] but its efficiency, as we shall see later, reaches Carnot limit $\eta_{C}$ only under the open circuit condition or when current and output power vanish.

Thermal gradients in conducting systems induce electric currents. In the appropriately engineered structures they may be used to effectively generate pure spin or spin polarized currents. The experimental demonstration of the possibility [6] has sparked a renewed interest in thermoelectric devices for applications in the emerging field of spintronics [7-11]. In this field it is important not only to obtain spin currents, but to have pure spin flow not accompanied by the charge flow. Dubi and Di Ventra's proposal [12] to get pure spin current in a device consisting of a quantum dot in an external $B$ field has stimulated a great deal of work in that direction $[13,14]$. The model has been generalized to the system which could sustain pure spin current without external magnetic field at the expense of using magnetic molecule instead of quantum $\operatorname{dot}[14]$.

More complicated systems and geometries were proposed and shown to offer new possibilities. Such structures include inter alia planar systems [15-20] or systems containing single or double quantum dots. Theoretical studies of double quantum dots have been started very early with the main interests being focused on the Coulomb blockade effect [21-26], shot noise, tunnel magnetoresistance $[27,28]$, etc. Later on, similar systems consisting of a single or double quantum dot have been extensively studied with the aim to find configurations with enhanced Seebeck coefficient $S_{c}$ or thermoelectric figure of 
merit $Z_{c} T[13,29-34]$ or their spin counterparts i.e. spin Seebeck coefficient $S_{s}$ and spin thermoelectric figure of merit $Z_{s} T[12,13,29,35-49]$.

It has been found inter alia that the thermoelectric figure of merit may be increased by Coulomb [50] and interference effects as well as direct hopping between the dots [51-55]. Impressive numerous experiments on the double quantum dots have to be noted [56-59] including those reporting the observation of the Kondo effect [60-62]. The early experimental and theoretical achievements have been summarized by van der Wiel and collaborators [63].

In this work we consider the system slightly different from those analyzed before. Our model describes nanostructure which may consist of two quantum dots coupled electrostatically to each other or a single dot with two levels. It is important to underline that if the central system is composed of two single level quantum dots it has a new degree of freedom which is related to independent tuning of the resonant levels. The freedom may be utilized e.g. for the efficient generation of the pure spin current. Moreover, the direction of the spin current is controlled electrically by appropriate gate voltage.

We study thermally induced charge, spin and heat flow in the sequential tunneling limit. In this limit the master equation approach to calculate the currents is adequate [64]. As already announced, we also calculate the performance of the system as an energy harvesting device. Our aim is to check if the nano-device with high value of $Z T$ performs really so well as expected from formula (1). According to the recent advances in the field one is mainly interested in the efficiency of the device at the finite power [65]. However, to gain additional insights into the performance of the system and its dependence on parameters we shall calculate both the efficiency and the power output for varying positions of the dot's resonant energies and load voltages.

The rest of the paper is organized as follows. In Section 2 we define the Hamiltonian of the model and briefly describe our approach to calculate charge, spin and heat currents. The linear response kinetic coefficients are defined in Section 3 and presented together with the thermoelectric figures of merit as a function of gate voltages. This allows identification of the regions with huge values of $Z T$ and large value of $\eta_{Z T}$ for subsequent comparision with $\eta$ calculated outside the linear regime. The definition of the efficiency of our device as a heat to electric power converter is introduced in Section 4 where the calculated efficiencies are also presented and discussed. The paper is concluded by a summary.

\section{The model and approach}

The system with interacting quantum dot(s) tunnel coupled to two external electrodes is described by the Hamiltonian

$$
H=H_{e l}+H_{D}+H_{T}
$$

where

$$
H_{e l}=\sum_{\mathbf{k}, \nu=L, R} \sum_{\sigma=\uparrow, \downarrow}\left(\varepsilon_{\nu \mathbf{k} \sigma}-\mu_{\nu}\right) c^{\dagger}{ }_{\nu \mathbf{k} \sigma} c_{\nu \mathbf{k} \sigma}
$$

is the energy operator for the external electrodes (left - L and right $-\mathrm{R}$ ). The spin dependence of the quasiparticle energies $\varepsilon_{\nu \mathbf{k} \sigma}$ applies to the ferromagnetic electrodes.

$$
\begin{aligned}
H_{D}= & \sum_{i=1,2} \sum_{\sigma=\uparrow, \downarrow} \varepsilon_{d i \sigma} d^{\dagger}{ }_{i \sigma} d_{i \sigma}+U_{1} n_{1 \uparrow} n_{1 \downarrow}+U_{2} n_{2 \uparrow} n_{2 \downarrow} \\
& +\sum_{\sigma, \sigma \prime} U_{12} n_{1 \sigma} n_{2 \sigma \prime}
\end{aligned}
$$

describes electrons on the quantum $\operatorname{dot}(\mathrm{s})$, and

$$
H_{T}=\sum_{i=1,2} \sum_{\mathbf{k}, \nu=L, R} \sum_{\sigma=\uparrow, \downarrow}\left(\gamma_{i \nu \mathbf{k} \sigma} c^{\dagger}{ }_{\nu \mathbf{k} \sigma} d_{i \sigma}+\text { H.c. }\right)
$$

is the tunneling Hamiltonian with, in general, spin dependent tunneling amplitudes. The operators $d_{i \sigma}^{\dagger}\left(d_{i \sigma}\right)$ create (annihilate) electrons of spin $\sigma$ on the $\operatorname{dot} i$, while $c_{\nu \mathbf{k} \sigma}^{\dagger}\left(c_{\nu \mathbf{k} \sigma}\right)$ create (annihilate) spin $\sigma$ electrons in the state $\mathbf{k}$ of the electrode $\nu . n_{i \sigma}=d_{i \sigma}^{\dagger} d_{i \sigma}$ is the number operator counting electrons on the $i$ th dot in a two dot geometry or $i$ th level of the two level dot. The $\sigma$ dependence of the ondot energy $\varepsilon_{i d \sigma}$ is due to the external magnetic field acting on the spins only, i.e. we neglect any orbital effect of $\mathbf{B}$ field on the leads; $\varepsilon_{d i \sigma}=\varepsilon_{d i}+g_{\sigma} \mu_{B} B, g_{\uparrow}=1, g_{\downarrow}=-1$. The effective couplings between the $i$ th dot (level) and $\nu$ th electrode $\Gamma_{i, \nu}^{\sigma}=\sum_{\mathbf{k}}\left|\gamma_{i \nu \mathbf{k} \sigma}\right|^{2} \delta\left(\varepsilon-\varepsilon_{\nu \mathbf{k} \sigma}\right)$ is energy independent in the wide band limit. The couplings in general depend on the spin of the tunneling electrons. This reflects the combined effect of spin dependent density of states in the ferromagnetic electrodes and possible spin dependence of the tunneling matrix elements $\gamma_{i \nu \mathbf{k} \sigma}$.

Denoting the probability to find quantum dot in a state $|n\rangle$ by $P_{n}$, the charge current between the $\nu$ th electrode and a quantum dot is given by [66]

$$
I_{c}^{\nu}=-e \sum_{n m}\left(W_{n m}^{\nu(\mathrm{in})}-W_{n m}^{\nu(\mathrm{out})}\right) P_{m}
$$

where the transition rates are calculated by means of the Fermi golden rule and the tunnelling Hamiltonian (5) we obtain

$$
W_{m n}^{\nu(\text { out })}=\frac{2 \pi}{\hbar} \sum_{\nu_{\mathbf{k}}, \mathbf{k} \sigma} \rho_{\nu_{\mathbf{k}}}\left|\left\langle m\left|c_{k \sigma} H_{T}^{\nu}\right| n\right\rangle\right|^{2} \delta\left(\varepsilon_{\nu \mathbf{k} \sigma}-E_{m}+E_{n}\right)
$$

for the tunnelling of an electron from the dot onto the $\nu$ th lead which changes the initial state of the dot from $n$ to $m$. Similar expression is obtained for a tunnelling of an electron from $\nu$ th lead onto the central region

$$
W_{m n}^{\nu(\mathrm{in})}=\frac{2 \pi}{\hbar} \sum_{\nu_{k}, \mathbf{k}, \sigma} \rho_{\nu_{\mathbf{k}}}\left|\left\langle m\left|c_{k \sigma}^{+} H_{T}^{\nu}\right| n\right\rangle\right| \delta\left(\varepsilon_{\nu \mathbf{k} \sigma}+E_{m}-E_{n}\right) .
$$


$\rho_{\nu_{\mathbf{k}}}$ denotes the elements of the density matrix of the lead and we explicitly indicated initial and final states of the dot only.

In the regime of sequential tunneling, the probabilities $P_{n}$ are calculated from the master equation [66]

$$
\frac{d P_{n}}{d t}=-\sum_{m \neq n} W_{m n} P_{n}+\sum_{m \neq n} W_{n m} P_{m},
$$

where the symbols $W_{n m}\left(W_{m n}\right)$ denote the total transition rates from initial state $m(n)$ to final state $n(m)$. They are sums of the (in) and (out) rates for all leads. For calculating the currents we need the probabilities in the stationary limit $\frac{d P_{n}}{d t}=0$.

The total transition rates $W_{m n}$ are sums of rates for a given direction of spin $\sigma$ and for each level (or dot) $i$ and electrode $\nu$ : $W_{m n, i \sigma}^{\nu}$. They allow us to calculate the spin dependent currents flowing in each of the electrodes via a particular level (or dot) $I_{i \sigma}^{\nu}$ :

$$
I_{i \sigma}^{\nu}=\sum_{m n} \sigma W_{m n, i \sigma}^{\nu} P_{m}
$$

In the most general case of two quantum dots there are 16 different states $n$ of the central region and the same number of probabilities $P_{n}$. Master equation for them becomes $16 \times 16$ matrix equation which in the general case we solve numerically.

\section{Linear response}

The main aim of this section is to calculate linear transport coefficients of the device at hand and define the regimes in which the expected value of $Z T$ is large. This will be used in the next section to compare efficiency $\eta_{Z T}$ expected from equation (1) and the actual efficiency of the device operating as the energy harvester.

Having obtained various contributions to the currents $I_{i \sigma}^{\nu}$ we define the spin dependent current through the system $I_{\sigma}$ and total charge $I_{c}$ and spin $I_{s}$ currents

$$
\begin{aligned}
& I_{c}=I_{\uparrow}+I_{\downarrow}, \\
& I_{s}=I_{\uparrow}-I_{\downarrow} .
\end{aligned}
$$

In a similar way one calculates and defines heat current $I_{Q}$ flowing in the system. Again denoting heat current carried by spin $\sigma$ electrons as $I_{Q, \sigma}$ one defines total heat flow as the sum $I_{Q}=I_{Q, \uparrow}+I_{Q, \downarrow}$. In the linear response the above fluxes may be written in terms of thermodynamic forces $e \Delta V=\Delta \mu=\mu_{L}-\mu_{R}, \Delta \mu_{\sigma}=\mu_{L \sigma}-\mu_{R \sigma}$ and $\Delta T / T$ (with $\Delta T=T_{L}-T_{R}$ ) and kinetic coefficients [13] $\mathcal{L}_{i j}^{\sigma}$

$$
\begin{aligned}
I_{\sigma} & =\frac{e}{h}\left(\mathcal{L}_{11}^{\sigma} \Delta \mu_{\sigma}+\mathcal{L}_{12}^{\sigma} \frac{\Delta T}{T}\right) \\
I_{Q, \sigma} & =\frac{1}{h}\left(\mathcal{L}_{21}^{\sigma} \Delta \mu_{\sigma}+\mathcal{L}_{22}^{\sigma} \frac{\Delta T}{T}\right) .
\end{aligned}
$$

The above equations are valid for systems with long spin relaxation rates in electrodes. Under non-equilibrium conditions the distribution of electrons in the leads is assumed to be given by the Fermi-Dirac function but in the system with long spin relaxation time the spin dependent chemical potentials $\mu_{\alpha \sigma}$ and the temperatures $T_{\alpha \sigma}$ have to be assumed. In this work, however, we neglect the last possibility and assume spin independent temperatures. Under this condition one may define [13] the spin voltage (or spin bias) $e \Delta V_{s}=\left(\Delta \mu_{\uparrow}-\Delta \mu_{\downarrow}\right) / 2$ and the (charge) voltage $e \Delta V=\left(\Delta \mu_{\uparrow}+\Delta \mu_{\downarrow}\right) / 2$.

In systems with short spin relaxation times the spin voltage $V_{s}$ vanishes and one writes standard equations [67]

$$
\begin{aligned}
I_{c} & =\frac{e}{h}\left(\mathcal{L}_{11} \Delta \mu+\mathcal{L}_{12} \frac{\Delta T}{T}\right) \\
I_{Q} & =\frac{1}{h}\left(\mathcal{L}_{21} \Delta \mu+\mathcal{L}_{22} \frac{\Delta T}{T}\right) .
\end{aligned}
$$

Charge $G_{c}$ and spin $G_{s}$ conductances are expressed in terms of $\mathcal{L}_{11}^{\sigma}$ as $G_{c}=\mathcal{L}_{11}^{\uparrow}+\mathcal{L}_{11}^{\downarrow}$ and $G_{s}=\mathcal{L}_{11}^{\uparrow}-\mathcal{L}_{11}^{\downarrow}$, respectively, while the thermopower $S$ is given by

$$
S=\frac{k_{B}}{e} \frac{\mathcal{L}_{12}}{k_{B} T \mathcal{L}_{11}},
$$

with $\mathcal{L}_{i j}=\sum_{\sigma} \mathcal{L}_{i j}^{\sigma}$. Thermal conductivity $\kappa$ is defined as a coefficient between the heat current $I_{Q}$ and temperature difference under the condition of no charge flow $\left(I_{c}=0\right)$

$$
\kappa=-\left(\frac{I_{Q}}{\Delta T}\right)_{I_{c}=0},
$$

and in the linear response is given by

$$
\kappa=\frac{\mathcal{L}_{22} \mathcal{L}_{11}-\mathcal{L}_{12} \mathcal{L}_{21}}{T \mathcal{L}_{11}} .
$$

The Peltier coefficient $\Pi$ is directly related to the Seebeck coefficient $S$. In bulk systems the Peltier effect manisfests itself as heating or cooling at the biased junction between two different conductors. In a nanostructure another interpretation of the Peltier coefficient $\Pi$ is more insightfull. It represents the amount of heat carried over the junction per unit charge and is defined as the ratio between heat and charge fluxes in the system with voltage but not temperature bias $(V \neq 0$ but $\delta T=0)$

$$
\Pi=\left(\frac{I_{Q}}{I}\right)_{\delta T=0}=\frac{\mathcal{L}_{21}}{\mathcal{L}_{11}}=S T .
$$

Efficient energy conversion devices are based on the Peltier effect for cooling and the Seebeck effect for power generation [68].

To find the currents and kinetic coefficients we first calculate all the rates $W_{m n}$ and the transition probabilities $P_{n}$ with an obvious normalization $\sum_{n} P_{n}=1$. Then the currents are given by the formulas (6) and (10). For simplicity we put the chemical potentials of the leads to their common value $\left(\mu_{L}=\mu_{R}=0\right)$, which is our zero of energy. We shall measure temperature $T$ and magnetic field $B$ in energy units and energies in the units of $\Gamma_{L}=\Gamma$. This means we assume $k_{B}=1$ and $\mu_{B}=1$. 

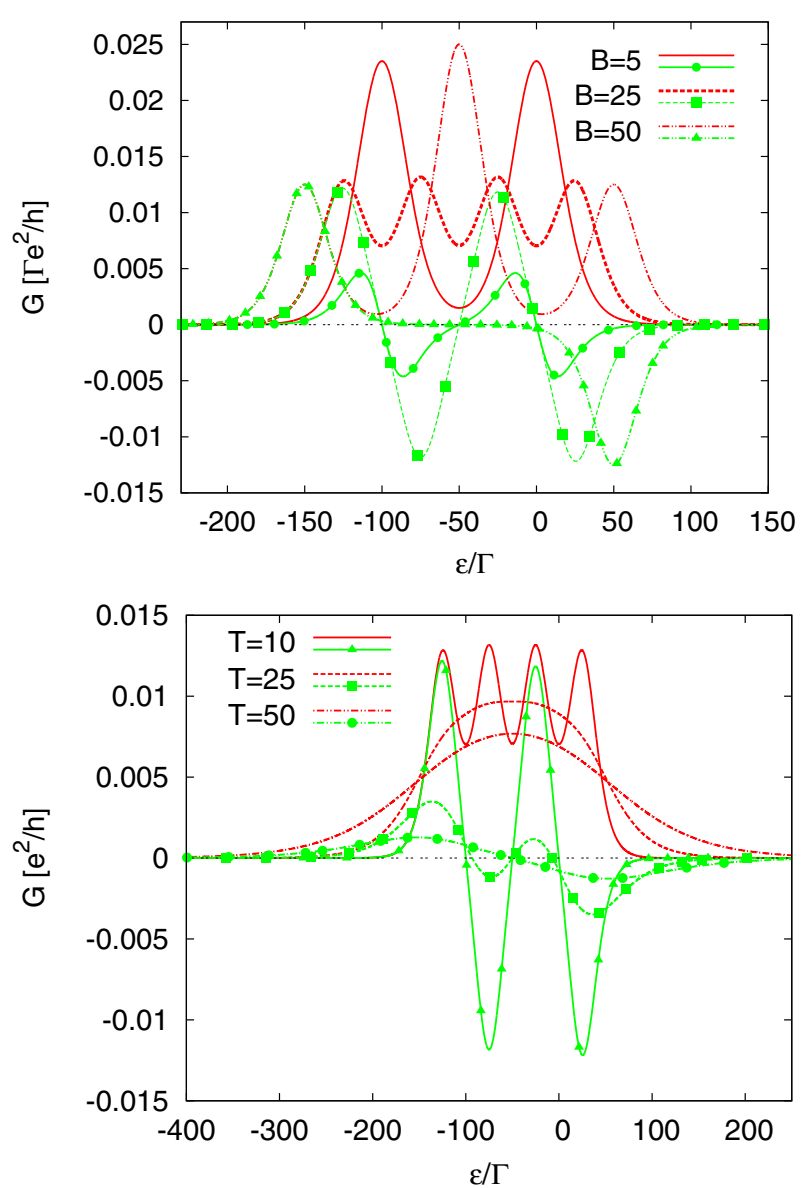

Fig. 1. The dependence of the charge and spin (curves with symbols) conductivity on the gate voltage for two level noninteracting $\left(U_{1}=U_{2}=U_{12}=0\right)$, symmetrically coupled $\left(\Gamma_{R}=\Gamma_{L}\right)$ quantum dot with the level splitting $\delta \varepsilon=100 \Gamma_{L}$ in an external magnetic field of varying strength (upper panel) at $T=10 \Gamma_{L}$ and for $B=25 \Gamma_{L}$ and three different temperatures (lower panel).

The results for charge and spin conductivity have been shown in Figure 1. Upper panel of this figure illustrates the dependence of conductivities on the gate voltage. The model we use is the two level dot with the distance between levels $\delta \varepsilon=100 \Gamma$. Three sets of curves in the left panel show conductances for temperature $T=10 \Gamma$ and three different values of the magnetic field. For very small magnetic fields $B=5 \Gamma$ one observes two broadened peaks in charge conductance centered around the values of gate voltage corresponding to two levels of the quantum dot. Spin splitting is not resolved for the magnetic field and temperature values. Higher magnetic fields $(B=25 \Gamma)$ split each of the levels and the $G(\varepsilon)$ gets more complicated with four peaks. For $B=50 \Gamma$ spin down peak of the upper level coincides with spin up one of the lower level giving three peak structure.

The corresponding behavior of the spin current is also easy to understand, as in the magnetic field spin down electrons have energies slightly lower than those with spin up. It means that for bias voltage lower than that corre- sponding to the resonant one, a single direction of the spin dominates, while above that value opposite spin electrons mainly contribute to the current. As a result spin current changes sign each time resonant level crosses the Fermi energy. The competition between spin up and spin down electrons for $B=50 \Gamma$ is most severe close to particle-hole symmetry point and it results in the relatively low spin current and conductance.

The influence of temperature on charge and spin conductances of the same system is shown in the lower panel of Figure 1 for moderate value of the magnetic field $B=25 \Gamma$. Interestingly, while increase of the temperature to $T=25 \Gamma$ smears out any structure in the gate dependence of charge conductance it affects mainly the amplitude of the spin conductance. At high temperatures of the order of spin-split level spacings the effect on the spin conductance is stronger and only outer peaks of spin conductance survive. Their amplitude, however, is strongly diminished as illustrated by thin line with stars. Even at these high temperatures the current is spin polarized and the direction of polarization can be changed by the gate voltage.

Another view of the presented results can be obtained by different look at the resulting expressions for the currents. In close analogy to the Landauer description valid for non-interacting systems the spin dependent charge current across the system may be (for symmetric couplings) approximately written as

$$
I_{c}^{\sigma}=\sum_{i} \int d E T_{i, \sigma}^{e f f}(E)\left[f_{L}(E)-f_{R}(E)\right],
$$

with $T_{i, \sigma}^{e f f}(E) \propto \Gamma_{i \sigma} \delta\left(E-\varepsilon_{i, \sigma}^{e f f}\right)$ denoting the energy dependent transmission across the system with $\Gamma_{i \sigma}$ being an appropriate coupling between the dot (level) and the electrodes. The energies $\varepsilon_{i, \sigma}^{e f f}$ are eigenvalues of the effective dot Hamiltonian and depend on $\varepsilon_{d 1 \sigma}, \varepsilon_{d 2 \sigma}$ and the interaction parameters $U_{1}, U_{2}$ and $U_{12}$. The total signal is a sum of all contributions, which may overlap. The difference of the Fermi functions for small bias voltage $\mu_{L}-\mu_{R}=e V$ and small temperature bias $\Delta T=T_{L}-T_{R}$ can be expanded to linear leading order. Then the conductance $G^{\sigma}$ is written as

$$
G^{\sigma}(\varepsilon) \propto \frac{e^{2}}{h} \sum_{i} \Gamma_{i \sigma}\left(-\frac{\partial f(\varepsilon)}{\partial \varepsilon}\right)_{\varepsilon=\varepsilon_{i, \sigma}^{e f f}} \frac{1}{k_{B} T} .
$$

To understand the Seebeck coefficient $S$, which is a voltage needed to stop the current flow induced by temperature bias $\delta T$ we quote the relation, known as Mott-Cutler (MC) formula, which gives $S$ in term of conductance [69] at the Fermi energy $G\left(\varepsilon_{F}\right)$

$$
S_{M C}=-\frac{\pi^{2}}{3} \frac{k_{B}}{e} k_{B} T \frac{1}{G\left(\varepsilon_{F}\right)}\left(\frac{d G(\varepsilon)}{d \varepsilon}\right)_{\varepsilon_{F}} .
$$

In the present case it leads to

$$
S \propto \frac{k_{B}}{e} \frac{\sum_{i, \sigma} \Gamma_{i \sigma}\left(-\frac{\partial f(\varepsilon)}{\partial \varepsilon}\right)_{\varepsilon=\varepsilon_{i, \sigma}^{e f f}} \frac{\varepsilon_{i, \sigma}^{e f f}-\mu}{k_{B} T}}{\sum_{i, \sigma} \Gamma_{i \sigma}\left(-\frac{\partial f(\varepsilon)}{\partial \varepsilon}\right)_{\varepsilon=\varepsilon_{i, \sigma}^{e f f}}} .
$$



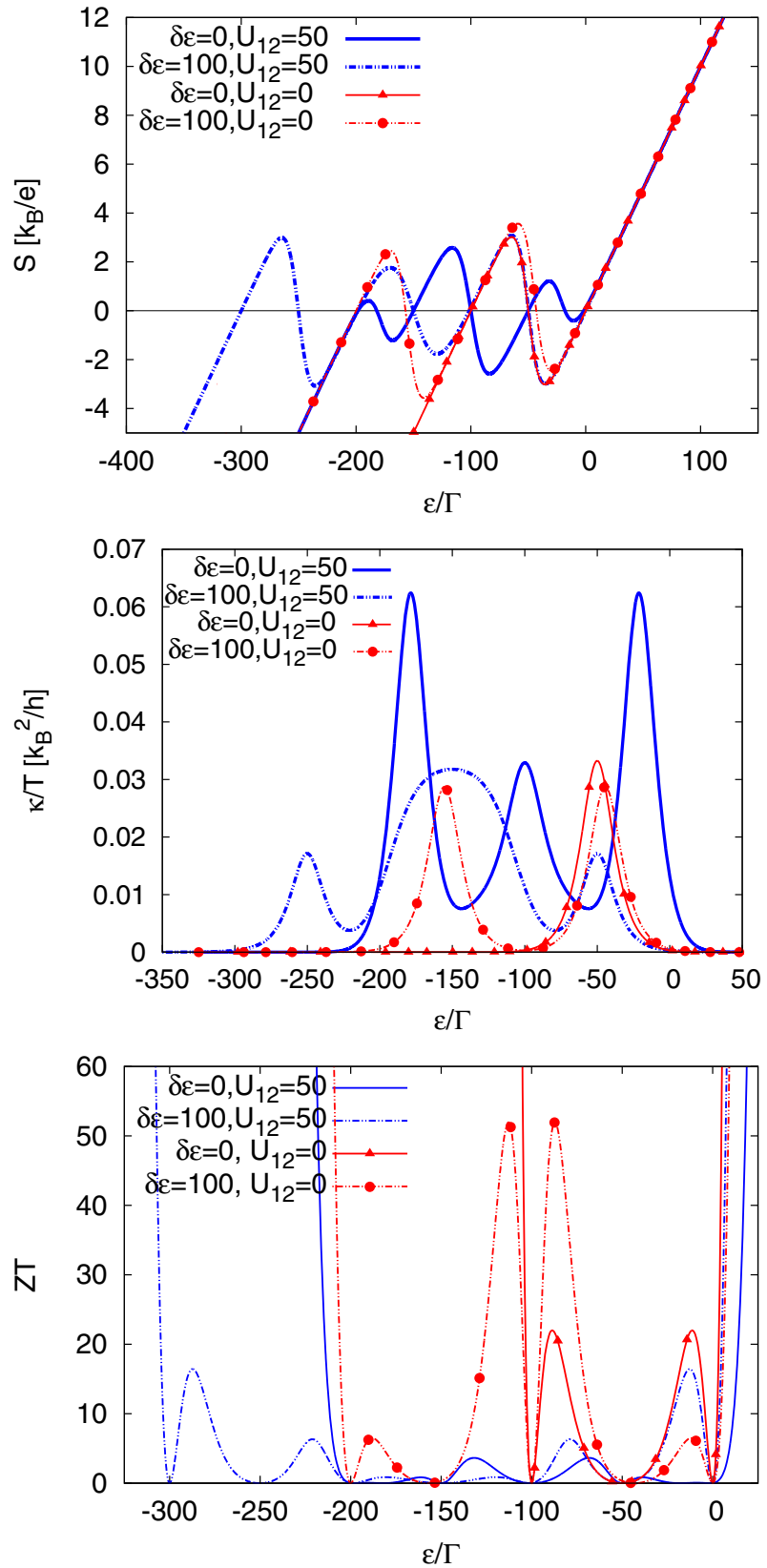

Fig. 2. The gate voltage dependence of the charge thermoelectric power (upper panel), thermal conductivity (middle panel) and thermoelectric figure of merit $Z T$ (lower panel) for two quantum dots with equally tuned levels $\varepsilon_{d 1}=\varepsilon_{d 2}=\varepsilon$ and two level quantum dot with $\delta \varepsilon=\varepsilon_{d 1}-\varepsilon_{d 2}=100 \Gamma_{L}$, intralevel interactions $U=100 \Gamma$, interlevel interaction $U_{12}=50 \Gamma$ at temperature $T=10 \Gamma_{L}$ and without magnetic field $B=0$.

The above equations show that the effective width of the conductance peak is given by the temperature $T$ and the thermopower $S$ changes sign each time chemical potential $\mu$ crosses one of the eigenvalues $\varepsilon_{i, \sigma}^{\text {eff }}$.

Figure 2 shows the gate voltage dependence of the thermoelectric power (upper panel), thermal conductivity (middle panel) and thermoelectric figure of merit $Z T$ (lower panel) for two models at temperature $T=10 \Gamma$.
One model is the double quantum dot with equally tuned energy levels $\varepsilon_{d 1}=\varepsilon_{d 2}=\varepsilon$, while the other is two level quantum dot with distance between levels $\delta \varepsilon=\varepsilon_{d 1}-\varepsilon_{d 2}=$ $100 \Gamma$. We assume that both levels experience rigid shift by the gate voltage. In some cases one could expect different (albeit linear) dependence of different levels on the gate voltage [64]. Here we neglect such possibility. The Seebeck coefficient has a characteristic sawtooth shape. Thick continuous curve and the curve with stars refer to double quantum dot with energy levels changing with the gate voltage at the same speed. The curves illustrate the effect of intradot Coulomb repulsion $\left(U_{12}=0\right.$ or $\left.50 \Gamma\right)$. The repulsion $U_{12}$ modifies Seebeck coefficient as it introduces additional effective levels $\varepsilon_{i, \sigma}^{e f f}$ at the dot as discussed above. The other curves (that with dots and the dashed one) show the effect of interlevel Coulomb repulsion $U_{12}$ for two level dot with $\varepsilon_{d 1}-\varepsilon_{d 2}=\delta \varepsilon=100 \Gamma$.

The observed changes of $S$ and thermal conductance $\kappa$ reflect the appearance of new resonant levels $\varepsilon_{i, \sigma}^{\text {eff }}$ in the system with additional interaction. The lower panel of the figure shows gate dependence of $Z T$ for both models. It is important to note that $Z T$ takes on huge values well beyond those known for bulk systems. $Z T$ rises to even higher values beyond the gate voltages shown in the figure. However, as we shall see in the next section, this does not necessarily mean that the thermoelectric heat engine based on the dots will work with efficiency close to $\eta_{C}$.

\section{Quantum dot as a thermoelectric power generator}

As discussed in the introduction the large figure of merit $Z T$ (c.f. Eq. (1)) is used as an indicator for good thermoelectric performance of the material [3] or device. It means that for very large $Z T$ the efficiency $\eta_{Z T}$ approaches the Carnot value. For simplicity we start the discussion with the simple case of single level interacting quantum dot. For this system the parameter $Z T$ has been calculated analytically [5] and found to be

$$
\begin{aligned}
Z T= & \left(\frac{k_{B} T}{U}\right)^{2} e^{\frac{\varepsilon_{d}}{k_{B} T}} \\
& \times \frac{\left[\frac{\varepsilon_{d}}{k_{B} T}\left(1+2 e^{\frac{\varepsilon_{d}}{k_{B} T}}+e^{\frac{2 \varepsilon_{d}+U}{k_{B} T}}\right)+\frac{U}{k_{B} T}\left(1+e^{\frac{\varepsilon_{d}}{k_{B} T}}\right)\right]^{2}}{\left(1+e^{\frac{\varepsilon_{d}}{k_{B} T}}\right)\left(1+e^{\frac{\varepsilon_{d}+U}{k_{B} T}}\right)} .
\end{aligned}
$$

It diverges for non-interacting system $(U=0)$, meaning that the efficiency of the non-interacting quantum dot based engine is given by the Carnot value $\eta_{Z T}=\eta_{C}$.

Here we shall calculate the actual efficiency $\eta$ of the thermolectric setup with non-interacting quantum dot beyond linear regime. Imagine the unbiased device consisting of two leads and the quantum dot tunnel coupled to both of them. If one of the leads (say R) is hot and another cold the charge carriers move from the hot to the cold side of 

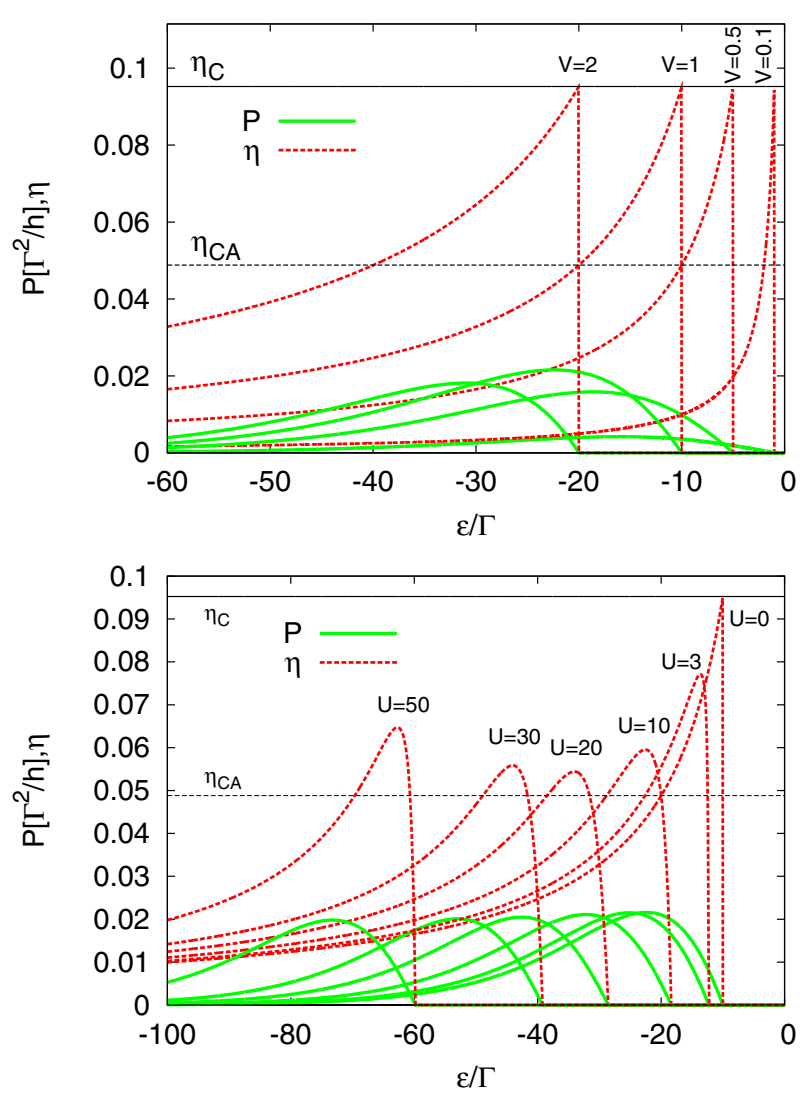

Fig. 3. The $\varepsilon$ dependence of the efficiency (dotted lines) and the power (full lines) of the single level quantum dot device in the region where it operates as the energy generator. The vertical lines join efficiencies and powers calculated for the same parameters. Upper panel is for non-interacting QD $(U=0)$, while the lower one shows the effect of interactions $U$ for $V=1 \Gamma$. Horizontal thin lines denoted $\eta_{C}$ and $\eta_{C A}$ show the theoretical estimates of the Carnot and Curzon-Ahlborn values of the efficiencies.

the device carrying both charge and energy. Under the open circuit condition the voltage builds in. The maximal voltage depends on the temperature difference and other parameters of the system. This voltage may be utilised to power the external circuit. Knowing the voltage and the temperature difference one calculates the charge and heat currents. This is enough to calculate the power $P$ delivered and the heat flux $\dot{Q}$ extracted from the hot electrode. One defines the efficiency of the setup as the ratio $\eta=P / \dot{Q}$ and calculates it for different values of the model parameters. The results for the noninteracting quantum dot are shown in Figure 3 (upper panel).

Calculating the performance characteristics of the device with quantum dots [70] we concentrate on the optimal conditions. We assume $T_{R}-T_{L}=\Delta T>0$ and $\mu_{L}-\mu_{R}=e V$. The efficiency may be written as

$$
\eta=-\frac{\dot{W}}{\dot{Q}}=\frac{V\left|I_{c}\right|}{I_{Q}^{R}} .
$$

In the two terminal device the charge current is conserved $I_{c}=I_{L}=-I_{R}$ and the heat current from the hot termi- nal $I_{Q}^{R}$ (under the external load) differs from that in the left terminal $I_{Q}^{L}$ as a result of internal energy (E) conservation. From thermodynamics we have

$$
\frac{d E}{d t}=\dot{Q}+\dot{W}=I_{Q}^{R}+I_{Q}^{L}+I_{c} V=0 .
$$

The system under study may work as an energy generator or as refrigerator depending on the region in its parameter space. Here we are interested in the energy harvesting. In the process the energy is extracted from the hot lead and transformed into electric power. The goal is to optimize the device for a given working temperatures.

Let us note that by fixing the working parameters of our device i.e. the couplings between the central region and external leads, temperature differences and the voltage we calculate heat currents $I_{Q}^{R}, I_{Q}^{L}$ and the charge current $I_{c}$. The calculated currents fulfill, as they should, equation (27). We make use of these fluxes in calculations of the efficiency of the device which is defined by equation (26).

The most important from practical point of view is the power delivered by the engine and its efficiency at that power [65]. The analysis of the performance of a simple non-interacting quantum dot generator [71] has shown the validity of the Curzon-Ahlborn prediction for the efficiency at maximum power

$$
\eta_{C A}=1-\sqrt{T_{1} / T_{2}}=1-\sqrt{1-\eta_{C}},
$$

where $\eta_{C}$ is the standard Carnot efficiency, while $T_{1}, T_{2}$ denote the working temperatures of the engine. Let note in passing that the analysis of endoreversible engines has become a hot topic nowadays. Many interesting results have been obtained for different nano-devices [71-75]. Some of the achievements have been reviewed recently [76].

In the upper panel of Figure 3 we present the efficiency $\eta$ (dotted lines) and the power $P=V I_{c}$ (full lines) as a function of $\varepsilon$ for the system with single level noninteracting quantum dot. The temperature of the hot lead $T_{R} / \Gamma=10.5$, the temperature difference $T_{R}-T_{L}=1 \Gamma$. The efficiency of the device as a function of the gate voltage is shown for four values of the load voltages $V / \Gamma=0.1,0.5,1.0,2.0$ (from right to left). The device works as an energy harvester in the region of $\varepsilon$ to the left from vertical lines. The vertical lines join efficiencies and powers calculated for the same set of parameters.

It easy to understand the result on physical grounds. Note, that for non-interacting quantum dot symmetrically coupled to external leads the charge and heat currents are simply given by

$$
\begin{aligned}
I_{c} & =-e \Gamma\left[f_{R}\left(\varepsilon_{d}\right)-f_{L}\left(\varepsilon_{d}\right)\right] \\
I_{Q}^{R} & =\Gamma\left(\varepsilon_{d}-\mu_{R}\right)\left[f_{R}\left(\varepsilon_{d}\right)-f_{L}\left(\varepsilon_{d}\right)\right] .
\end{aligned}
$$

The current vanishes for

$$
\frac{\varepsilon_{d}-\mu_{L}}{k_{B} T_{L}}=\frac{\varepsilon_{d}-\mu_{R}}{k_{B} T_{R}} .
$$

This allows to write the efficiency as

$$
\eta=\frac{\mu_{L}-\mu_{R}}{\varepsilon_{d}-\mu_{R}}
$$


or, for the symmetric bias $\mu_{L / R}=\mu \mp e V / 2$, to obtain the relation

$$
\varepsilon_{d}=\mu-\frac{e V}{2} \frac{T_{R}+T_{L}}{T_{R}-T_{L}}
$$

which means that for a given bias load $V$ and temperatures of the electrodes the system may be tuned by gate voltage (i.e. changing $\varepsilon_{d}$ ) to give maximal efficiency.

At the point when the condition (32) is fulfilled the current vanishes and the efficiency (31) reduces to $\eta=$ $\left(T_{R}-T_{L}\right) / T_{R}$, i.e. the Carnot value. Note that in the figures we have set the equilibrium value of the chemical potential to $\mu=0$. Using the relation between the heat $(Q)$ and entropy $(S)$ flows $I_{S}^{R, L}=I_{Q}^{R, L} / T_{R, L}$ it is easily shown that the entropy flow is not balanced except at the point when (30) is fulfilled. This proves the reversibility of the operation and justifies the Carnot value of the efficiency.

The points of reversible operation are marked in Figure 3 by the vertical lines for each set of parameters and denote the limiting (maximum) value of the gate voltage $(\varepsilon)$ for the device to operate as the energy generator. The requirement is that $I_{Q}^{R}>0$, i.e. the heat is extracted from the hot electrode.

At the point (32) the efficiency $\eta$ reaches the Carnot limit $\eta_{C}=1-\frac{T_{L}}{T_{R}}=1-9.5 / 10.5 \approx 0.0952$ and the power vanishes as it should for the reversibly operating engine. For a given voltage load $V$, tuning the device left from the vertical line decreases its efficiency from the ideal value. At the same time the output power (full lines in the figure) increases. It takes on maximal value for some gate voltage and decreases again as a result of decreasing charge current in the system tuned far away from the resonant condition. This detuning is a result of large negative bias $\varepsilon$ when the dot level moves well below the equilibrium value of the chemical potential $\mu=0$.

The efficiency and maximum power output of the interacting quantum dot change with the system parameters in a more complicated way (see the lower panel of Fig. 3). With increasing $U$ from $U=0$ the maximum efficiency decreases from the Carnot value, goes over minimum and increases again towards the Carnot limit with increasing $U$. At the limits of $U \rightarrow 0$ and very large $U \rightarrow \infty$ resonant levels $\left(\varepsilon_{d}+U\right)$ effectively disappear from the transport window, thus opening the doors to the large values of the efficiency close to $\eta_{C}$. For each set of parameters the gate voltage $\varepsilon$ is used to tune the system (whether interacting or not) to maximal efficiency or maximal output power. This general behavior is the same for all voltage loads. The non-monotonic dependence of the maximal efficiency on the intra-dot interaction has been previously established and analyzed in great details by Muralidharan and Grifoni [70] in a related study.

The maximum output power depends on the voltage load, gate voltage and other parameters of the system. In the figures the changes of $P$ are relatively less visible than that of efficiency. As a function of voltage bias $\varepsilon$ the behaviour is the following. The power starts from the zero value goes over maximum and decreases again. Looking at the maximum power output as a function of load voltage $V$ one observes (c.f. Fig. 3 - upper panel) its increase up to the largest value and later decrease for $V$ larger than optimal one. For some set of parameters the output power attains global maximum.

The general behaviour of both efficiency and the output power as seen in the lower panel of Figure 3 may be understood by noticing that in the case of interacting quantum dot the transport goes via two energy levels $\varepsilon_{d}$ and $\varepsilon_{d}+U$. Similarily two levels contribute to transport in the model with two level quantum dot. The total charge and heat currents are sums of currents flowing via each of the levels, i.e. $I_{c, Q}=I_{c, Q}\left(\varepsilon_{1}\right)+I_{c, Q}\left(\varepsilon_{2}\right)$. The reversibility condition is never fulfilled for $\varepsilon_{1} \neq \varepsilon_{2}$ (or $U \neq 0$ ) and the efficiency is less than Carnot value for all parameters. However, due to the energy factors $\left(\varepsilon_{i}-\mu_{L, R}\right)$ in front of heat currents (29) the heat from the hot lead changes with gate voltage in a different way than the charge current and this results in the observed dependence of the efficiency and the power. The heat extracted from the right lead grows slower/faster than the charge current explaining increase/decrease of the power and efficiency. Note that for interacting system both $P$ and $\eta$ start from zero go through the maximum and fall down again at large detuning. The vanishing of $\eta$ and $P$ follow from the vanishing of charge current not accompanied by the simultaneous vanishing of heat current. For a very large interaction or the distance between levels one asymptoticaly reaches the single level limit.

Figure 4 (upper panel) shows the dependence of the efficiency (dotted curves) and power (full lines) on the gate voltage in a single level quantum dot for a few values of the magnetic field $B$. Note the systematic decrease of the maximum efficiency with $B$ field (in units of $\Gamma$ ) accompanied by the shift towards lower gate voltages. The maximum value of the power changes only slightly with $B$. This behaviour is easily understood as the main effect of the magnetic field is the spin splitting of the level. The system becomes effectively two level one and the previous arguments apply.

The lower panel of the figure shows the same characteristics for two dot system with equally tuned energy levels $\varepsilon_{d 1}=\varepsilon_{d 2}=\varepsilon$ for a few values of the interdot interaction $U_{12}$. The on-dot interactions are assumed to be equal $U_{1}=U_{2}=40 \Gamma$. The maximum efficiency (but not the efficiency at maximum power) is above the Curzon-Ahlborn limit $\eta_{C A}$ (marked by the horizontal dashed line) for small values of $U_{12}$ and falls below it for larger $U_{12}$. At constant load voltage the maximum value of the output power only weakly depends on the $U_{12}$.

The dependence of maximum $\eta$ on magnetic field as well as on interdot interactions illustrate the general rule that the presence of other energy levels in an energy window of a few $k_{B} T$ from a working level generally leads to its diminishing as discussed above.

The comparison of $\eta_{Z T}$ found from equation (1) using the calculated values of $Z T$ with $\eta$ calculated from definition (26) for the same setup is shown in Figure 5 for two different situations. Upper panel of that figure refers to the single level interacting quantum dot with $U=10 \Gamma$. Again it shows that the expected value of the efficiency 

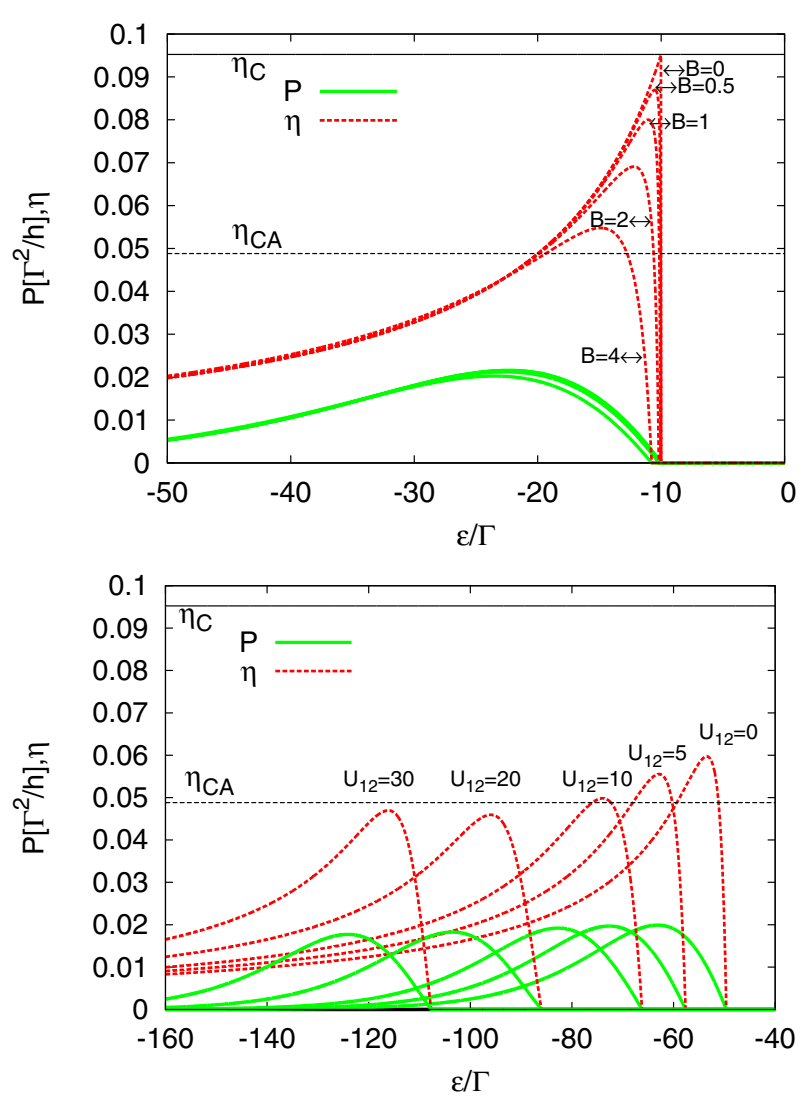

Fig. 4. The dependence of the efficiency and the power on the gate voltage. In the upper panel the voltage load $V=1 \Gamma$ and we show results for a few values of magnetic field $B$ applied to single level non-interacting quantum dot. Lower panel shows the effect of the interdot interaction $U_{12}$ on two dot system with $\varepsilon_{1}=\varepsilon_{2}=\varepsilon_{d}, U_{1}=U_{2}=40 \Gamma$ and the same load voltage.

is different from the actual one for most regions in the parameter space. Moreover for the actual efficiency of the device to be maximum the system has to be appropriately tuned. The maximum efficiency expected on the basis of equation (1) differs from that calculated for the device. The devices with many levels contributing to the transport are characterised by the complicated pattern of regions when they may be used as energy harvesters as seen in the lower panel of the figure. The mere knowledge of $Z T$ is not enough to decide when the device works as an energy harvester and if it performs optimally. Different load voltages $V$ require slightly different tuning to have maximum power. The tuning is easily done by electrical means (gate voltage).

As mentioned earlier our model allows the study of a setup with two independently tuned quantum dots. We show the maps of $\eta$ and $P$ on the $\left(\varepsilon_{1}, \varepsilon_{2}\right)$ plane in Figure 6. For some parameters the efficiency attains values close to the Carnot value. This corresponds to the cases of effective decouplings of two levels. In the white regions of the parameter space the device operates as a heat pump.

Close inspection of two panels in Figure 6 shows that the maximum of the power lies in slightly different places in parameter space in comparison with efficiency. Also the
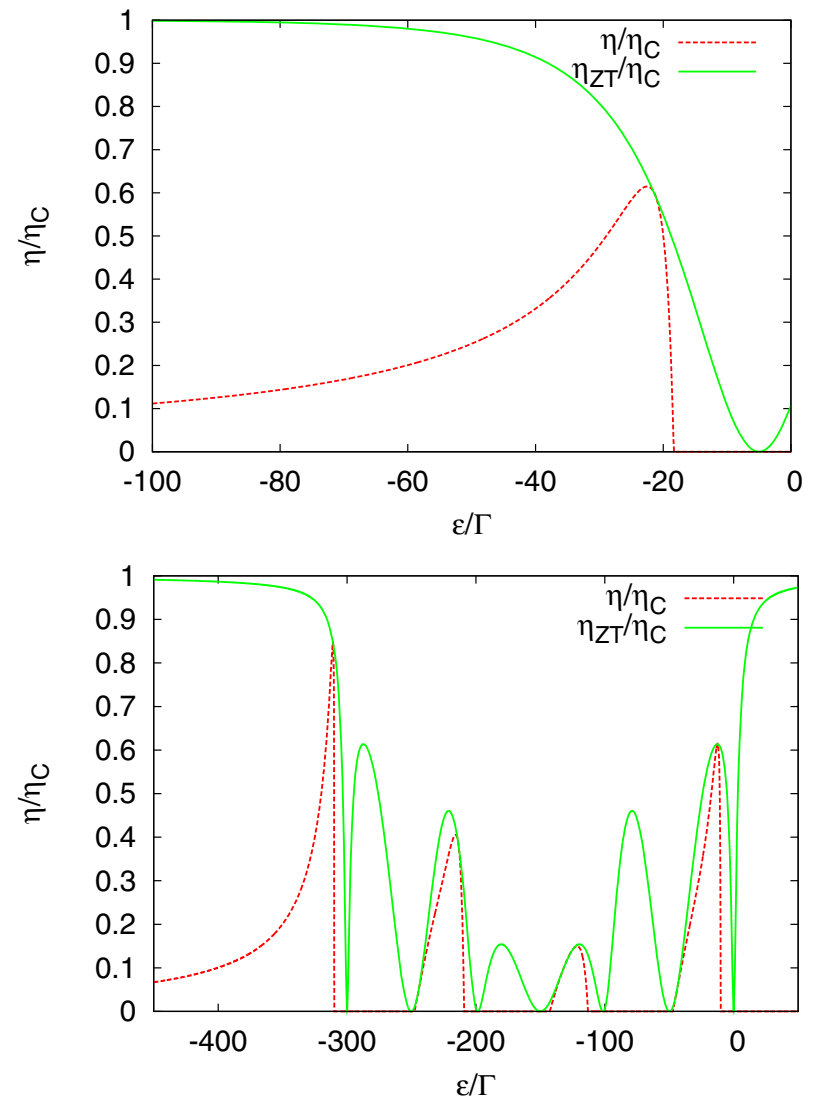

Fig. 5. The dependence on the gate voltage of the expected efficiency $\eta_{Z T}$ of the engine and the calculated efficiency $\eta$ both normalised to the Carnot value for $T=\left(T_{L}+T_{R}\right) / 2=10 \Gamma$, $\delta T=1 \Gamma$. Upper panel refers to the single level interacting quantum dot with $U=10 \Gamma$. The lower panel is for two level quantum dot with gate independent distance between the levels $\delta \varepsilon=100 \Gamma$, intralevel interaction $U=100 \Gamma$ and interlevel repulsion $U_{12}=50 \Gamma$ (c.f. Fig. 2 for $Z T$ of the same system).

power and efficiency of the two-dot two-terminal system are largest when both levels lie on the same side of the chemical potential (those were the system works as an energy harvesting engine).

\section{Summary and conclusions}

We have analyzed thermoelectric performance of the model which describes devices with the degenerate single level or two level quantum dot or two electrostatically coupled quantum dots. Two quantum dots can be tuned independently of each other. This feature distinguishes the system from the two level quantum dot. When the magnetic field is applied the system can be tuned to the state with pure spin current. Spin current can be induced by means of external voltage or temperature difference between the electrodes.

Transport coefficients including charge and spin conductances and thermoelectric figure of merit [77] have been calculated in the linear regime and the regions of very large enhancement of $Z T$ identified. We have also 

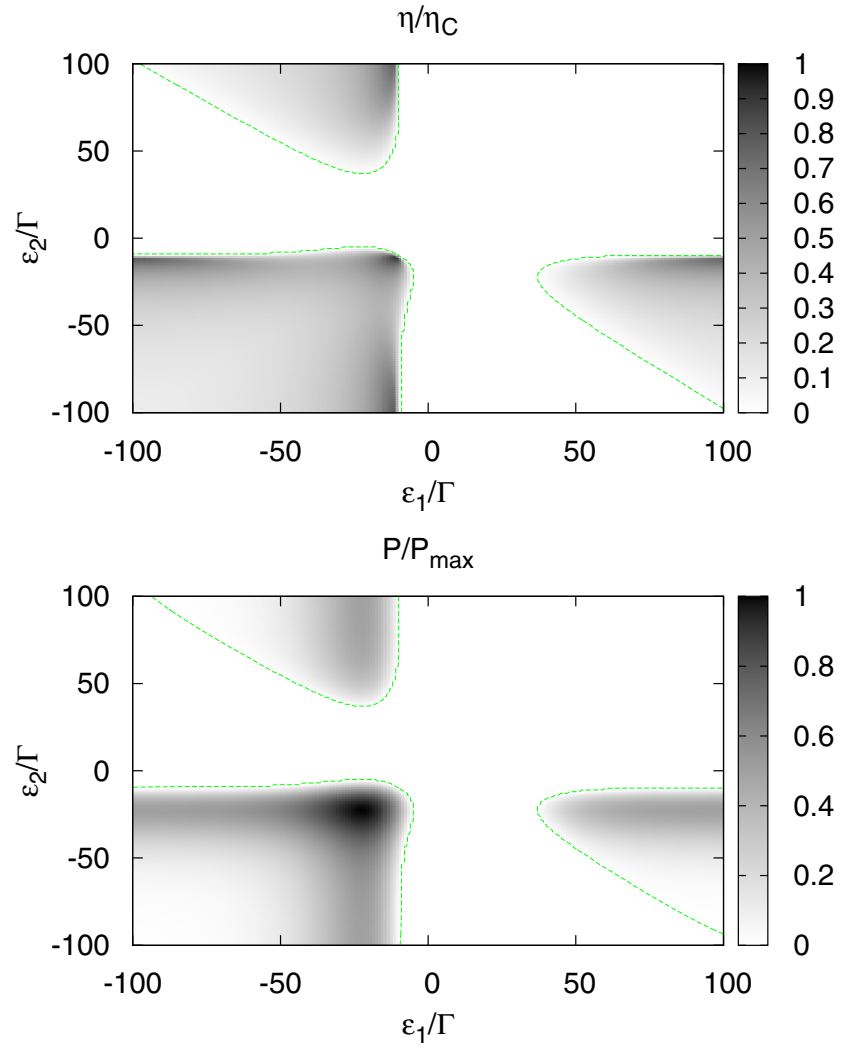

Fig. 6. The dependence of the efficiency $\eta$ normalized to the Carnot value (upper panel) and the power normalized to its maximum value for a given voltage $V=1 \Gamma$ (lower panel) on the gate voltages $\varepsilon_{1}$ and $\varepsilon_{2}$ for two non-interacting quantum dots. Temperature $T=10 \Gamma$ and the temperature difference between hot and cold leads $\Delta T=1 \Gamma$.

calculated the output power and the efficiency of the device working as a converter of heat into electricity. The study shows that large values of $Z T$ do not necessarily mean the usefulness of the device as an energy harvester. In some parameter range it performs rather poorly, i.e. its output power is very small and the efficiency well below the Carnot limit even when $Z T$ is very large [70].

The efficiency of the noninteracting system may attain the Carnot value in the limit of the irreversible operation. Under that condition the power of the device vanishes in agreement with the general thermodynamic laws. We show how the efficiency and the power output change with the voltage load, the temperature difference and other model parameters. In particular, the on-dot and interdot/interlevel Coulomb interactions have strong and generally detrimental effect on the efficiency of the device under the constant load.

The maximum efficiency of the interacting system may exceed the Curzon-Ahlborn value for some parameters. However, it usually corresponds to the power output slightly smaller than the maximum one. Interestingly, the on-dot $U_{i}$ or interdot/interlevel $U_{12}$ interactions have rather small effect on the maximum value of the output power despite strong effect on the efficiency. For some values of parameters the calculated efficiency at maximum power slightly exceeds (not shown) the Curzon-Ahlborn value. This remains in agreement with the previous study of Esposito et al. [71].

This work has been partially supported by the National Science Centre under the contract DEC-2011/01/B/ST3/04428.

\section{References}

1. J.P. Heremans, C.M. Thrush, D.T. Morelli, Phys. Rev. B 70, 115334 (2004)

2. J.P. Heremans, Acta Phys. Pol. A 108, 609 (2005)

3. G.D. Mahan, J.O. Sofo, Proc. Natl. Acad. Sci. 93, 7436 (1996)

4. A.I. Boukai, Y. Bunimovich, J. Tahir-Kheli, J.-K. Yu, W.A. Goddard III, J.R. Heath, Nature 451, 168 (2008)

5. P. Murphy, S. Mukerjee, J. Moore, Phys. Rev. B 78, 161406 (2008)

6. K. Uchida, S. Takahashi, K. Harii, J. Ieda, W. Koshibae, K. Ando, S. Maekawa, E. Saitoh, Nature 455, 778 (2008)

7. T. Dietl, in Semiconductor Spintronics, Lecture Notes in Physics (Springer, Berlin, 2007), Vol. 712, pp. 1-46

8. W.J.M. Naber, S. Faez, W.G. van der Wiel, J. Phys. D 40, $205(2007)$

9. J. Fabian, A. Matos-Abiague, C. Ertler, P. Stano, I. Zutic, Acta Phys. Slovaca 57, 565 (2007)

10. I. Zutic, J. Fabian, S. Das Sarma, Rev. Mod. Phys. 76, $323(2004)$

11. L. Bogani, W. Wernsdorfer, Nat. Mater. 7, 179 (2008)

12. Y. Dubi, M. Di Ventra, Phys. Rev. B 79, 081302 (2009)

13. R. Świrkowicz, M. Wierzbicki, J. Barnaś, Phys. Rev. B 80, 195409 (2009)

14. R.-Q. Wang, L. Sheng, R. Shen, B. Wang, D.Y. Xing, Phys. Rev. Lett. 105, 057202 (2010)

15. S. Russo, M. Kroug, T.M. Klapwijk, A.F. Morpurgo, Phys. Rev. Lett. 95, 027002 (2005)

16. J.L. Webb, B.J. Hickey, G. Burnell, Phys. Rev. B 86, $054525(2012)$

17. P. Machon, M. Eschrig, W. Belzig, Phys. Rev. Lett. 110, 047002 (2013)

18. M.M. Wysokiński, Acta Phys. Pol. A 122, 758 (2012)

19. M.M. Wysokiński, J. Spalek, J. Appl. Phys. 113, 163905 (2013)

20. J. Ren, J. Fransson, J.-X. Zhu, Phys. Rev. B 89, 214407 (2014)

21. L.I. Glazman, V. Chandrasekhar, Europhys. Lett. 19,623 (1992)

22. R. Ziegler, C. Bruder, H. Schoeller, Phys. Rev. B 62, 1961 (2000)

23. V.N. Golovach, D. Loss, Phys. Rev. B 69, 245327 (2004)

24. T. Hayashi, T. Fujisawa, H.D. Cheong, Y.H. Jeong, Y. Hirayama, Phys. Rev. Lett. 91, 226804 (2003)

25. L. Oroszlany, A. Kormanyos, J. Koltai, J. Cserti, C.J. Lambert, Phys. Rev. B 76, 045318 (2007)

26. B.R. Bułka, T. Kostyrko, M. Tolea, I.V. Dinu, J. Phys.: Condens. Matter 19, 255211 (2007)

27. F. Bodoky, W. Belzig, C. Bruder, Phys. Rev. B 77, 035302 (2008)

28. I. Weymann, Phys. Rev. B 78, 045310 (2008)

29. M. Wierzbicki, R. Swirkowicz, Phys. Rev. B 84, 075410 (2011)

30. P. Trocha, J. Barnaś, Phys. Rev. B 85, 085408 (2012) 
31. M.B. Tagani, H.R. Soleimani, Solid State Commun. 152, $914(2012)$

32. M.B. Tagani, H.R. Soleimani, J. Appl. Phys. 112, 103719 (2012)

33. C. Jiang, W.J. Gong, Y.S. Zheng, Eur. Phys. J. B 85, 364 (2012)

34. G. Rajput, P.K. Ahluwalia, K.C. Sharma, Physica B 406, $3328(2011)$

35. T. Rejec, R. Żitko, J. Mravlje, A. Ramŝak, Phys. Rev. B 85, $085117(2012)$

36. X.F. Yang, Y.S. Liu, J. Appl. Phys. 113, 164310 (2013)

37. X.K. Hong, Y.S. Liu, J.F. Feng, J.H. Chu, J. Appl. Phys. 114, 144309 (2013)

38. F. Chi, J. Zheng, X.-D. Lu, K.-C. Zhang, Phys. Lett. A 375, 1352 (2011)

39. S. Lipiński, D. Krychowski, Phys. Rev. B 81, 115327 (2010)

40. J.Y. Luo, H.J. Jiao, B.T. Xiong, X.-L. He, C. Wang, J. Phys.: Condens. Matter 25, 155304 (2013)

41. L. Ai-Xian, D. Su-Qing, Chin. Phys. B 21, 117201 (2012)

42. D.-K. Fang, S.-Q. Wu, C.-Y. Zou, G.-P. Zhao, Chin. Phys. Lett. 29, 037303 (2012)

43. W. Qiang, X. Hai-Qing, J. Hu-Jun, L. Zhi-Jian, N. YiHang, Chin. Phys. B 21, 117310 (2012)

44. W.-J. Gong, C. Jiang, X. Sui, A. Du, J. Phys. Soc. Jpn 81, 104601 (2012)

45. J. Zheng, F. Chi, Y. Guo, J. Phys.: Condens. Matter 24, 265301 (2012)

46. J. Azema, A.-M. Dare, S. Schäfer, P. Lombardo, Phys. Rev. B 86, 075303 (2012)

47. G. Gomez-Silva, O. Avalos-Ovando, M.L. Ladron de Guevara, P.A. Orellana, J. Appl. Phys. 111, 053704 (2012)

48. Z.C. Yang, Q.F. Sun, X.C. Xie, J. Phys.: Condens. Matter 26, $045302(2014)$

49. A. Ozaeta, P. Virtanen, F.S. Bergeret, T.T. Heikkila, Phys. Rev. Lett. 112, 057001 (2014)

50. N.A. Zimbovskaya, J. Chem. Phys. 140, 104706 (2014)

51. I. Weymann, P. Trocha, Phys. Rev. B 89, 115305 (2014)

52. F. Chi, J. Zheng, X.-D. Lu, K.-C. Zhang, Phys. Lett. A 375, 1352 (2011)

53. J.Y. Luo, H.J. Jiao, Y. Shen, G. Cen, X.-L. He, C. Wang, J. Phys.: Condens. Matter 23, 145301 (2011)

54. J.A. Andrade, P.S. Cornaglia, A.A. Aligia, Phys. Rev. B 89, $115110(2014)$

55. S. Sharmin, K. Muraki, T. Fujisawa, Phys. Rev. B 89, 115315 (2014)

56. M.R. Graber, M. Weiss, D. Keller, S. Oberholzer, C. Schonenberger, Ann. Phys. 16, 672 (2007)

57. M.R. Graber, W.A. Coish, C. Hoffmann, M. Weiss, J. Furer, S. Oberholzer, D. Loss, C. Schonenberger, Phys. Rev. B 74, 075427 (2006)
58. M. Pioro-Ladriere, R. Abolfath, P. Zawadzki, J. Lapointe, S.A. Studenikin, A.S. Sachrajda, P. Hawrylak, Phys. Rev. B 72, 125307 (2005)

59. L. DiCarlo, H.J. Lynch, A.C. Johnson, L.I. Childress, K. Crockett, C.M. Marcus, M.P. Hanson, A.C. Gossard, Phys. Rev. Lett. 92, 226801 (2004)

60. S.J. Chorley, M.R. Galpin, F.W. Jayatilaka, C.G. Smith, D.E. Logan, M.R. Buitelaar, Phys. Rev. Lett. 109, 156804 (2012)

61. S. Amasha, A.J. Keller, I.G. Rau, A. Carmi, J.A. Katine, H. Shtrikman, Y. Oreg, D. Goldhaber-Gordon, Phys. Rev. Lett. 110, 046604 (2013)

62. A.J. Keller, S. Amasha, I. Weymann, C.P. Moca, I.G. Rau, J.A. Katine, H. Shtrikman, G. Zarnd, D. GoldhaberGordon, Nat. Phys. 10, 145 (2013)

63. W.G. van der Wiel, S. De Franceschi, J.M. Elzerman, T. Fujisawa, S. Tarucha, L.P. Kouwenhoven, Rev. Mod. Phys. 75, 1 (2002)

64. D.A. Bagrets, Yu.V. Nazarov, Phys. Rev. B 67, 085316 (2003)

65. F.L. Curzon, B. Ahlborn, Am. J. Phys. 43, 22 (1974)

66. H. Bruus, K. Flensberg, in Many-body Quantum Theory in Condensed Matter Physics (Oxford University Press, New York, 2004), Chap. 10

67. G.D. Mahan, in Many-Particle Physics (Plenum Press, New York, London, 1981), Chap. 3.8

68. G. Chen, M.S. Dresselhaus, G. Dresselhaus, J.-P. Fleurial, T. Caillat, Int. Mater. Rev. 48, 45 (2003)

69. M. Cutler, N.F. Mott, Phys. Rev. 181, 1336 (1969)

70. B. Muralidharan, M. Grifoni, Phys. Rev. B 85, 155423 (2012)

71. M. Esposito, K. Lindenberg, C. Van den Broeck, Eur. Phys. Lett. 85, 60010 (2009)

72. M. Esposito, R. Kawai, K. Lindenberg, C. Van den Broeck, Phys. Rev. Lett. 105, 150603 (2010)

73. K. Yazawa, A. Shakouri, J. Appl. Phys. 111, 024509 (2012)

74. G. Benenti, G. Casati, J. Wang, Phys. Rev. Lett. 110, 070604 (2013)

75. N. Nakpathomkun, H.Q. Xu, H. Linke, Phys. Rev. B 82, 235428 (2010)

76. U. Seifert, Rep. Prog. Phys. 75, 126001 (2012)

77. M. Krawiec, K.I. Wysokiński, Phys. Rev. B 73, 075307 (2006)

Open Access This is an open access article distributed under the terms of the Creative Commons Attribution License (http://creativecommons.org/licenses/by/4.0), which permits unrestricted use, distribution, and reproduction in any medium, provided the original work is properly cited. 